\title{
Study on Wet Etching of AAO Template
}

\author{
Guofeng Hu, Haiming Zhang, Wenwen Di \& Tingting Zhao \\ School of Science, Tianjin Polytechnic University, Tianjin 300160, China \\ E-mail: hugf2009@163.com
}

\begin{abstract}
The highly ordered anodic aluminum oxide (AAO) template was prepared with two-step anodization. The AAO template was carried out wet etching process using a mixture of phosphoric acid and chromic acid, $\mathrm{NaOH}$, and $\mathrm{H}_{3} \mathrm{PO}_{4}$ solutions. The relationship between mass loss of AAO template and etching time was studied. The results indicate that 3 wt $\% \mathrm{H}_{3} \mathrm{PO}_{4}$ solution is a more mild and effective etchant comparing with these etchants, and the mass loss is linear with etching time. SEM investigations show that alumina nanowires were obtained with high yield by etching AAO template. And, the growth of alumina nanowires was closely related to the etching time.
\end{abstract}

Keywords: Porous Anodic aluminum oxide, Wet etching, $\mathrm{H}_{3} \mathrm{PO}_{4}$, Alumina nanowires

\section{Introduction}

Recently, One-dimensional (1 D) nanomaterials have stimulated great attention due to their optical and electronic properties. The template-assisted method has played an important role in the field of fabrication of $1 \mathrm{D}$ nanomaterials. The AAO template has uniform pore sizes, a high pore density, and a high aspect ratio (Masuda, 1995, pp. 1466-1468). These structural features of AAO template are controlled readily by appropriate choice of anodizing solutions, voltage or current density, solution temperature, and pore widening ( $\mathrm{Li}, 1998$, pp. 6023-6026). The AAO template has become an ideal template for these advantages. Nanowires or nanotubes of a variety of materials including metals (Sauer, 2002, pp. 3243-3247) (Sarkar, 2007, pp. 271-290), semiconductor materials (Yang, 2004, pp. 65-68) (Seo, 2007, pp. 241-244), and polymer materials (Steinhart, 2002, pp. 1997) (Pan, 2007, pp. 015302-015307), have been fabricated by using AAO template. Carbon nanotubes have also been created in the AAO nanopores (Hu, 2001, pp. 3083-3085). But the nanodevices combined with AAO template are rare in the literature. The nanowires or nanotubes fabricated using AAO template have a highly ordered structure and a high aspect ratio. But when the AAO template was removed, the nanowires or nanotubes are easy to fall down together or form clusters for their high surface tension. This phenomenon has adversely affected the fabrication of nanodevices (Pan, 2005, pp. 1559-1564).

Therefore, the quantificational etching of AAO template has become a very urgent task. There will be a great breakthrough of fabrication of nanodevices based on AAO template, on condition that the length of exposed nanowires or nanotubes could be precise controlled by making from partial removal of AAO template via wet etching process. To obtain the highly ordered nanoarrays is of great significance in the fabrication of gas sensors and field emission devices by keeping the nanowire or nanotube arrays upright with the support of remaining AAO template (Hwang, 2005, pp. 850-858).

To date, the controllable wet chemical etching to remove partial AAO template is rare in the literature. And, the aqueous $\mathrm{NaOH}$ solution, the most common etchant, is available for etch AAO template (Chen, 2007, pp. 306-310) (Shankar, 2004, pp. 1312-1316). But the reaction between $\mathrm{NaOH}$ and AAO template always is too severe to control the progress of wet etching process. In this paper, we report a much milder wet etching of AAO template using the common $\mathrm{H}_{3} \mathrm{PO}_{4}$ solution. The wet etching effect by different etchants has been studied to achieve quantificational etching of AAO template.

\section{Experiment}

\subsection{Porous aluminum oxide template preparation}

High-purity aluminum foils (99.999\%) were employed in our experiment to fabricate the AAO template. The Al foil was first annealed at $500{ }^{\circ} \mathrm{C}$ for $2 \mathrm{~h}$, degreased using acetone and alcohol, then rinsed in distilled water. Al foil was electropolished in a mixed solution of $\mathrm{HClO}_{4}: \mathrm{C}_{2} \mathrm{H}_{5} \mathrm{OH}=1: 4(\mathrm{~V} / \mathrm{V})$ for 5 min to provide a smooth surface, then promptly rinsed with distilled water. Afterwards, the clean $\mathrm{Al}$ foil was anodized at $40 \mathrm{~V}$ DC voltage in $0.3 \mathrm{M}$ oxalic acid solution. 
After $6 \mathrm{~h}$ anodization, the sample was immersed in a mixture of phosphoric acid and chromic acid to remove the porous aluminum oxide formed in the first anodization. The remaining $\mathrm{Al}$ foil was anodized for a second time under the same conditions for $8 \mathrm{~h}$. Then the sample was etched by $\mathrm{CuCl}_{2}$ solution to remove the aluminum substrate.

\subsection{Process of wet etching of AAO template}

A number of clean and dry AAO templates which were removed aluminum substrate were weighted and immersed in different etchants respectively, until completely dissolved at room temperature. During the etching process, the AAO templates were taken out of etchants at regular intervals, rinsed with distilled water, dried and weighted. The etchants were used in our experiment including mixture of phosphoric acid and chromic acid $\left(6 \mathrm{wt} \% \mathrm{H}_{3} \mathrm{PO}_{4}\right.$ and 1.8 wt $\%$ $\left.\mathrm{H}_{2} \mathrm{CrO}_{4}\right), 3 \mathrm{M} \mathrm{NaOH}, 3$ wt $\% \mathrm{H}_{3} \mathrm{PO}_{4}, 5$ wt $\% \mathrm{H}_{3} \mathrm{PO}_{4}$, and $10 \mathrm{wt} \% \mathrm{H}_{3} \mathrm{PO}_{4}$.

The effect of wet etching of AAO template was studied with use of FESEM (LEO 1530VP).

\section{Results and Discussion}

\subsection{Wet etching of AAO template}

The component of AAO template is amorphous alumina. It is an amphoteric oxide which has reactions as both a base and an acid. The chemical equations for the etching reaction are as follows.
$2 \mathrm{OH}^{-}+\mathrm{Al}_{2} \mathrm{O}_{3}$
$\longrightarrow 2 \mathrm{AlO}_{2}^{-}+\mathrm{H}_{2} \mathrm{O}$
$6 \mathrm{H}^{+}+\mathrm{Al}_{2} \mathrm{O}_{3}$
$\longrightarrow 2 \mathrm{Al}^{3+}+3 \mathrm{H}_{2} \mathrm{O}$

It can be seen from Figure 1. that the reaction rate was rapid when AAO template was etched in $\mathrm{NaOH}$ solution. The AAO template was thinned rapidly, curled, and disappear in solution. The wet etching process in $3 \mathrm{M} \mathrm{NaOH}$ solution lasted only $7 \mathrm{~min}$. It is difficult to control the etching process of AAO template to obtain the appropriate length of exposed nanoarrays at such a rapid etching rate. In addition, $3 \mathrm{M} \mathrm{NaOH}$ could be available for a fast reagent to release nanostructures embedded in AAO template for its rapid dissolution rate of alumina.

Figure 2. shows the etching curve of AAO template in the mixture solution of phosphoric acid and chromic acid. The AAO template needs about $180 \mathrm{~min}$ to be completely dissolved. And the etching rate is obviously slower comparing to the rapid etching rate in $\mathrm{NaOH}$ solution.

Figure 3. shows the etching curves of AAO template in 3 wt $\% \mathrm{H}_{3} \mathrm{PO}_{4}, 5$ wt $\% \mathrm{H}_{3} \mathrm{PO}_{4}$, and $10 \mathrm{wt} \% \mathrm{H}_{3} \mathrm{PO}_{4}$. These curves are similar. The mass loss changed little within the first $30 \mathrm{~min}$, then increased rapidly with time and was linear with etching time. It may be due to the AAO template used in our experiment without removing barrier layer, phosphoric acid reacted with the barrier layer at first, and the etching rate is slow at the beginning. After approximately $30 \mathrm{~min}$, the pores of AAO template were opened, it allows more etchant permeated into AAO template free from the open pores, accelerated and made the etching rate linear.

There's no doubt that the etching rate is connected with the active etching component $\mathrm{H}^{+}$in $\mathrm{H}_{3} \mathrm{PO}_{4}$ solution, and the etching rate is proportional to its concentration. From the wet etching studies, the etching rate in $10 \mathrm{wt} \% \mathrm{H}_{3} \mathrm{PO}_{4}$ is higher than that in $5 \mathrm{wt} \% \mathrm{H}_{3} \mathrm{PO}_{4}$, both of which are higher than the etching rate in $3 \mathrm{wt} \% \mathrm{H}_{3} \mathrm{PO}_{4}$. The etching time of AAO template in $10 \mathrm{wt} \% \mathrm{H}_{3} \mathrm{PO}_{4}, 5 \mathrm{wt} \% \mathrm{H}_{3} \mathrm{PO}_{4}$, and $3 \mathrm{wt} \% \mathrm{H}_{3} \mathrm{PO}_{4}$ are $180 \mathrm{~min}, 330 \mathrm{~min}$ and $360 \mathrm{~min}$, respectively. And, the etching rate of $10 \mathrm{wt} \% \mathrm{H}_{3} \mathrm{PO}_{4}$ is similar to that in mixture of phosphoric acid and chromic acid. During the wet etching process, the AAO templates remained the robust state as before, not curly. The slower etching rate enable the precise control of wet etching time to expose the length of nanoarrays, and the etching time in $3 \mathrm{wt} \% \mathrm{H}_{3} \mathrm{PO}_{4}$ is more proper.

We have discovered that AAO templates would become ultrathin, and would be curled near terminal time. This phenomenon is similar to that in $\mathrm{NaOH}$ solution. It is hard to measure so thin an AAO template.

\subsection{Characterization of AAO template and Alumina nanowires}

Topography of the AAO template is shown in SEM images in Figure 4. The AAO template was formed with highly ordered hexagonal arrangement of $50 \mathrm{~nm}$ in diameter and the distance between neighboring pores was about $100 \mathrm{~nm}$ by anodization. And the pore density can reach $10^{10} \mathrm{~cm}^{-2}$, which is suitable for fabrication of large-scale uniform nanowire arrays.

It is shown in Figure 5. that the effect of wet etching of AAO template in mixture solution of phosphoric acid and chromic acid. It is clearly seen that the fallen nanowires on AAO template are disorder and with a high aspect ratio. The upper section of AAO template after wet etching is alumina nanowires clusters and the lower section also is AAO template.

\subsection{The mechanism of fabrication of alumina nanowires}

The alumina nanowires would be produced on the AAO template after wet etching whatever the etchant is a base or an acid. We attribute the fabrication of alumina nanowires to reason that the etchant react with the pore wall of AAO 
template (Zhang, 2005, pp. 1254-1258). When the etchant solution diffuses into the pores, the pore wall are dissolved for the active etching component $\mathrm{H}^{+}$or $\mathrm{OH}^{-}$reacts with the pore wall. Figure 6 . shows that the fabrication procedure of the alumina nanowires in the wet etching process. The thinnest of pore wall of two neighboring pores were etched away at first. Since the juncture of three neighboring pores is the thickest portion of alumina, the wet etching will produce alumina nanowires. The nanowires could fall down together without the support of the pore wall.

In the middle of wet etching process, the fabrication of alumina nanowires results in the acceleration of etching rate. Meantime, the mass loss of AAO template shows good linearity with the etching time.

It is because the active etching components can diffuse into the pores from the opposite end of the pore after barrier layer removal. It would accelerate the dissolution of the pore wall in AAO template. On the one hand, the alumina nanowires have ultrahigh aspect ratio which will greatly increase surface area of AAO template, and they are easy to be dissolved by etchants. On the other hand, the new alumina nanowires are being produced continuously, while the old nanowires are being dissolved. The dynamic balance between the alumina nanowires production and dissolution make the etching rate steady.

\section{Conclusions}

We have studied the wet etching of AAO template using several etchants, including $\mathrm{H}_{3} \mathrm{PO}_{4}$ solutions, a mixture solution of phosphoric acid and chromic acid and $\mathrm{NaOH}$ solution. The $3 \mathrm{wt} \% \mathrm{H}_{3} \mathrm{PO}_{4}$ solution is the best etchants to precise etch AAO template, comparing $3 \mathrm{M} \mathrm{NaOH}$ solution. And, its wet etching effect indicates that the mass loss of AAO template is good linear with the etching time in the wet etching process. A large number of alumina nanowires have been produced on etchants use. It's a good method to fabricate alumina nanowires. Meanwhile, the production of nanowires has played an important part in keeping a linear relationship between the mass losses of AAO template and etching time.

\section{References}

Chen, D., Song, G. J., Peng, Z. (2007). Preparation of metal nanowire arrays with controllable length using a simple etching method. Journal of Functional Materials and Devices, 13, 306-310

Hu, W., Gong, D., Chen, Z. (2001). Growth of well-aligned carbon nanotube arrays on silicon substrates using porous alumina film as a nanotemplate. Applied Physics Letters, 79, 3083-3085

Hwang, S. K., Lee, J., Jeong, S. H. (2005). Fabrication of carbon nanotube emitters in an anodic aluminium oxide nanotemplate on a Si wafer by multi-step anodization. Nanotechnology, 16, 850-858

Li, A. P., Müller, F., and Birner, A. (1998). Hexagonal pore arrays with a 50-420 nm interpore distance formed by self-organization in anodic alumina. Journal of Applied Physics, 84, 6023-6026

Masuda, H., and Fukuda, K. (1995). Ordered metal nanohole arrays made by a two-step replication of honeycomb structures of anodic alumina. Science, 268, 1466-1468

Pan, C., Zhang, L., Zhu, J. (2007). Surface decoration of anodic aluminium oxide in synthesis of Nafion ${ }^{\circledR}{ }^{-} 115$ nanowire arrays. Nanotechnology, 18, 015302-015307

Pan, H., Sun, H., Poh, C. (2005). Single-crystal growth of metallic nanowires with preferred orientation. Nanotechnology, 16, 1559-1564

Sarkar, J., Khan, G. G., and Basumallick, A. (2007). Nanowires: properties, applications and synthesis via porous anodic aluminium oxide template. Bulletin of Materials Science, 30, 271-290

Sauer, G., Brehm, G., Schneider, S. (2002). Highly ordered monocrystalline silver nanowire arrays. Journal of Applied Physics, 91, 3243-3247

Seo, B. I., Shaislamov U. A., Ha, M. H. (2007). ZnO nanotubes by template wetting process. Physica E, 37, 241-244

Shankar, K. S., and Raychaudhuri, A. K. (2004). Growth of an ordered array of oriented manganite nanowires in alumina templates. Nanotechnology, 15, 1312-1316

Steinhart, M., Wendorff, J. H., Greiner, A. (2002). Polymer nanotubes by wetting of ordered porous templates. Science, 296, 1997

Yang, Y., Chen, J., Chen, H. (2004). Study of CdS nanowires deposited in silicon based porous alumina film by non-aqueous electrochemical deposition. Chinese Journal of Inorganic Chemistry. 20, 65-68

Zhang, L., Yao, S., Zhang, H. (2005). Preparation and fabrication mechanism of alumina nanowires. Acta Physico-chimica Sinica, 21, 1254-1258 


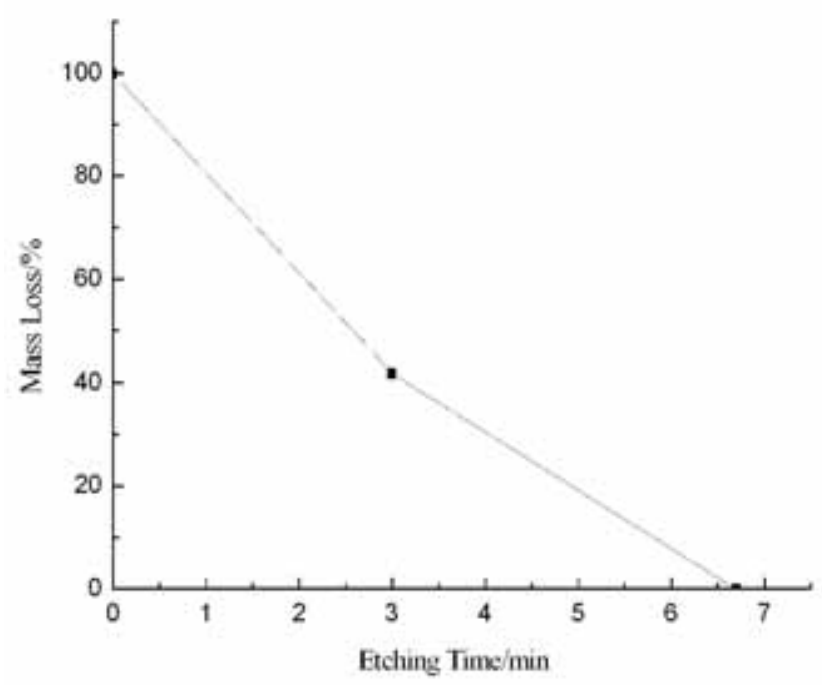

Figure 1. Wet Etching Curve of AAO Template in $3 \mathrm{M} \mathrm{NaOH}$

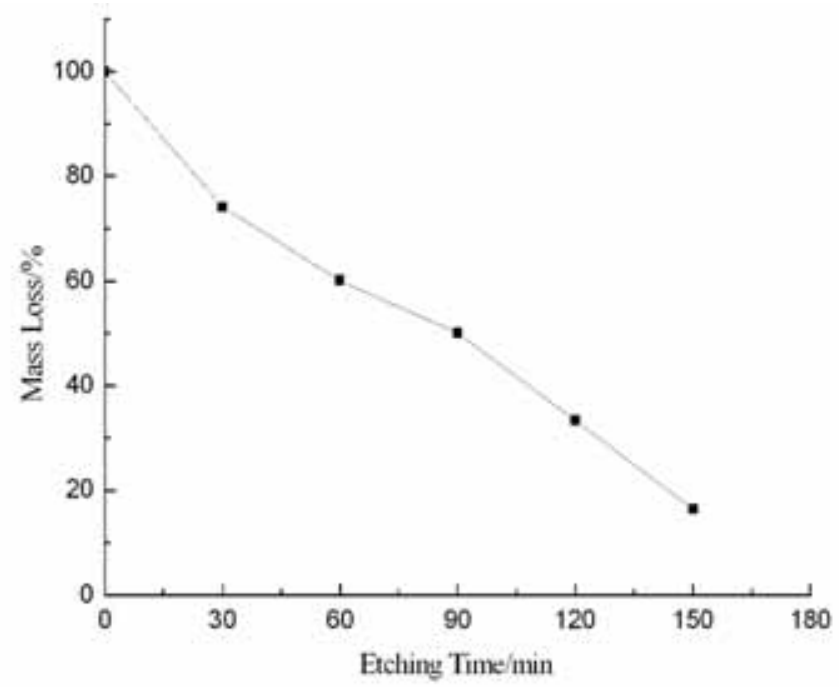

Figure 2. Wet Etching Curve of AAO Template in Mixture of $6 \mathrm{wt} \% \mathrm{H}_{3} \mathrm{PO}_{4}$ and $1.8 \mathrm{wt} \% \mathrm{H}_{2} \mathrm{CrO}_{4}$

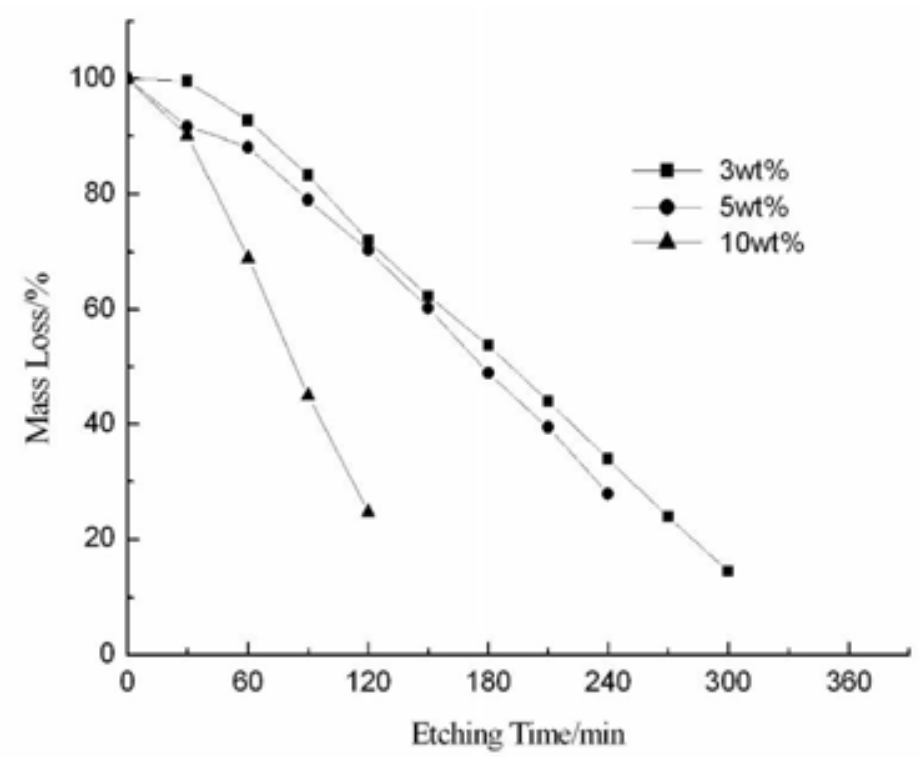

Figure 3. Wet Etching Curves of AAO Template in $\mathrm{H}_{3} \mathrm{PO}_{4}$ Solutions 


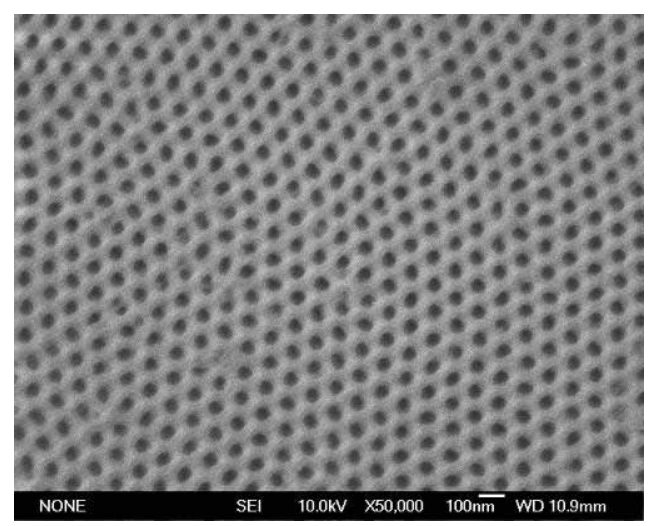

(a)

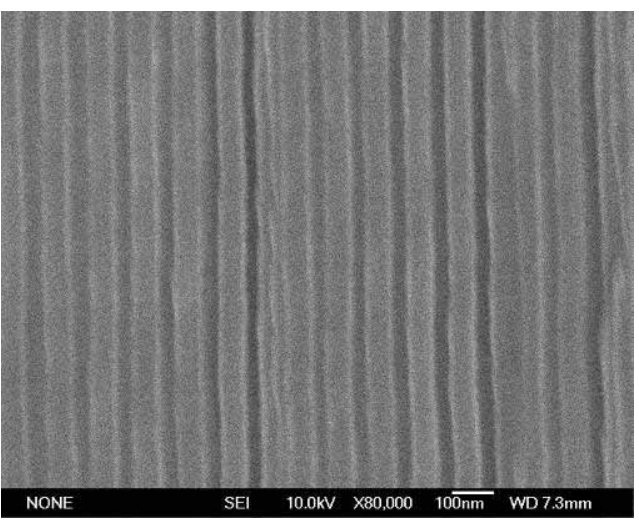

(b)

Figure 4. SEM Images of the AAO (a) Surface (b) Cross Section.

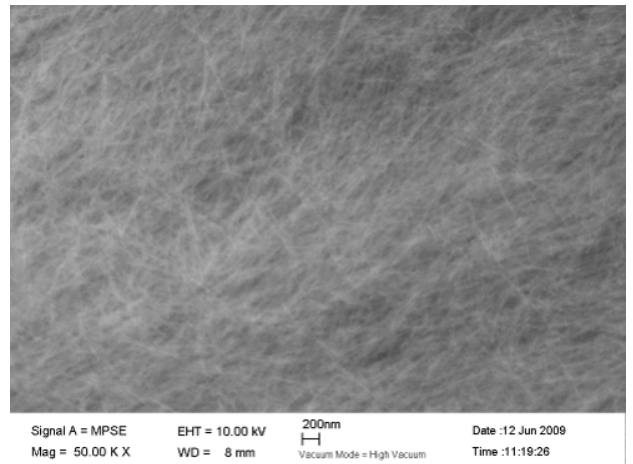

(a)

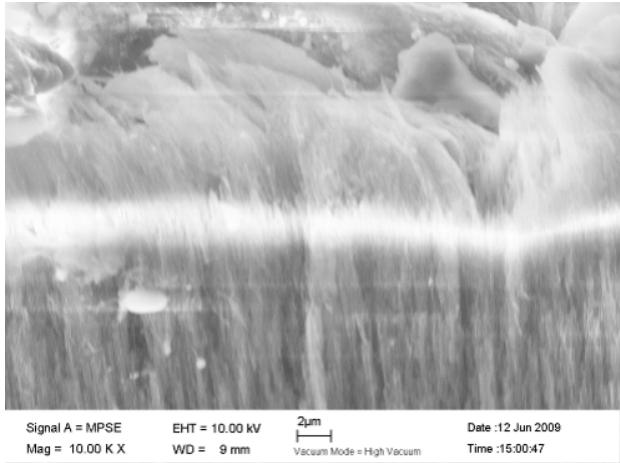

(b)

Figure 5. SEM Images of Alumina Nanowires on AAO Template (a) Surface (b) Cross Section.

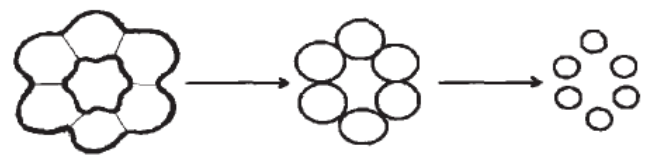

Figure 6. Schematic of Fabrication Procedure of the Alumina Nanowires 